VOL. 40 (1989) [425-428]

\title{
ON APPROXIMATION BY \\ TRIGONOMETRIC LAGRANGE INTERPOLATING POLYNOMIALS
}

\author{
T.F. XIE AND S.P. ZhOU
}

\begin{abstract}
It is well-known that the approximation to $f(x) \in C_{2 \pi}$ by $n$th trigonometric Lagrange interpolating polynomials with equally spaced nodes in $C_{2 \pi}$ has an upper bound In $(n) E_{n}(f)$, where $E_{n}(f)$ is the $n$th best approximation of $f(x)$. For various natural reasons, one can ask what might happen in $L^{p}$ space? The present paper indicates that the result about the trigonometric Lagrange interoplating approximation in $L^{p}$ space for $1<p<\infty$ may be "bad" to an arbitrary degree.
\end{abstract}

Let $L_{2 \pi}^{p}$ be the class of integrable functions of power $p$ and of period $2 \pi, C_{2 \pi}$ be the class of continuous $2 \pi$-periodic functions and $T_{n}$ be the trigonometric polynomials of degree at most $n$.

For $f \in L_{2 \pi}^{1}, S_{n}(f, x)$ is the $n$th partial sum of the Fourier series of $f(x)$; for $f \in$ $L_{2 \pi}^{p}, E_{n}(f)_{p}$, is the $n$th best approximation of $f(x)$ in $L^{p}$ space; for $f \in C_{2 \pi} L_{n}(f, x)$ is the $n$th trigonometric Lagrange interpolating polynomial of $f(x)$ with equally spaced nodes; that is

$$
L_{n}(f, x)=\sum_{k=0}^{2 n} f\left(x_{k}\right) l_{k}(x)
$$

where

$$
\begin{gathered}
l_{k}(x)=\frac{1}{2 n+1} \frac{\sin (n+1 / 2)\left(x-x_{k}\right)}{\sin 1 / 2\left(x-x_{k}\right)} \\
x_{k}=\frac{2 k \pi}{2 n+1}, \quad k=0,1, \ldots, 2 n
\end{gathered}
$$

The norm of $f \in L_{2 \pi}^{p}$ is defined as follows.

$$
\begin{gathered}
\|f\|_{L^{p}}=\left(\int_{0}^{2 \pi}|f(x)|^{p} d x\right)^{1 / p}, \quad 1 \leqslant p<\infty \\
\|f\|=\|f\|_{L^{\infty}}=\max _{0 \leqslant x \leqslant 2 \pi}|f(x)|, \quad p=\infty
\end{gathered}
$$

Received 4 January, 1989

'T'he second author would like to express his gratitude to Dr. P.B. Borwein for valuable discussions and suggestions which led to this revised manuscript.

Copyright Clearance Centre, Inc. Serial-fee code: 0004-9729/89 \$A2.00+0.00. 
It is well-known that

$$
\left\|S_{n}\right\|=\sup \left\{\left\|S_{n} f\right\|:\|f\|=1\right\} \sim \ln (n+1)
$$

which means that there exists a positive constant $M$ independent of $n$ such that

$$
M^{-1} \ln (n+1) \leqslant\left\|S_{n}\right\| \leqslant M \ln (n+1)
$$

so the factor $\ln (n+1)$ in the following inequality

$$
\left\|f-S_{n}(f)\right\|=0\left(\ln (n+1) E_{n}(f)\right) \text { for } f \in C_{2 \pi}
$$

cannot be omitted. However, in $L^{p}$ space for $1<p<\infty$, by the Riesz theorem (see [2]), a beautiful result is obtained for $f \in L_{2 \pi}^{p}$, namely:

$$
\left\|f-S_{n}(f)\right\|_{L^{p}} \leqslant c_{p} E_{n}(f)_{p}
$$

where $c_{p}$ is a positive constant depending only upon $p$. Below for convenience the symbol $c_{i}$ will denote a positive constant depending only upon at most $p$.

On the other hand, we can also see that (see [1])

$$
\left\|L_{n}\right\| \sim \ln (n+1)
$$

From (1) and (3), together with (2), it seems reasonable to guess that for $1<p<\infty$,

$$
\left\|f-L_{n}(f)\right\|_{L^{p}} \leqslant c_{p} E_{n}(f)_{p}, \quad f \in C_{2 \pi}
$$

Unfortunately, this is not true, as the following example shows.

Theorem. Let $1<p<\infty$ and let $\left\{\lambda_{n}\right\}$ be a positive decreasing sequence of real numbers such that $n^{s} \lambda_{n} \rightarrow 0$ for any $s>0$. Then there exists an infinitely differentiable function $f \in C_{2 \pi}$ such that

$$
\varlimsup_{n \rightarrow \infty} \frac{\left\|f-L_{n}(f)\right\|_{L^{p}}}{\lambda_{n}^{-1}\left\|f-S_{n}(f)\right\|_{L^{p}}}>0 .
$$

Lemma 1. Let $1<p \leqslant 2$. Then there exists a trigonometric polynomial $y_{n}(x)$ such that

$$
\begin{gathered}
\left\|g_{n}-L_{n}\left(g_{n}\right)\right\|_{L^{p}} \geqslant c \lambda_{n}^{-3} n^{1 / q}, \quad 1 / p+1 / q=1 \\
\left\|g_{n}-S_{n}\left(g_{n}\right)\right\|_{L^{p}}=0\left(\lambda_{n}^{-3 / 2} n^{1 / 2}\right)
\end{gathered}
$$


Proof: Set

$$
g_{n}(x)=\sum_{m=1}^{\left[\lambda_{n}^{-3}\right]} \sum_{k=m(2 n+1)}^{m(2 n+1)+n-1} \cos k x
$$

Since $\cos (m(2 n+1)+j) x_{k}=\cos j x_{k}$ for $0 \leqslant j \leqslant n-1$ and $0 \leqslant k \leqslant 2 n$, and $L_{n}(f, x) \in T_{n}, L_{n}\left(g_{n}, x\right)=\sum_{j=0}^{n-1}\left[\lambda_{n}^{-3}\right] \cos j x$. Applying the Hausdorff-Young inequality (see [2]) we have

$$
\left\|g_{n}-L_{n}\left(g_{n}\right)\right\|_{L^{p}} \geqslant c_{3}\left(\sum_{j=1}^{n-1}\left[\lambda_{n}^{-3}\right]^{q}\right)^{1 / q} \geqslant c_{4} \lambda_{n}^{-3} n^{1 / q}
$$

On the other hand $\left\|g_{n}-S_{n}\left(g_{n}\right)\right\|_{L^{p}}=\left\|g_{n}\right\|_{L^{p}} \leqslant c_{5}\left\|g_{n}\right\|_{L^{2}}$, so, from the Parseval equality, we have $\left\|g_{n}-S_{n}\left(g_{n}\right)\right\|_{L^{p}}=0\left(\lambda_{n}^{-3 / 2} n^{1 / 2}\right)$, and we have proved (4) and (5).

Similarly, with a slight change to $g_{n}(x)$, applying the Hölder inequality to $\| g_{n}-$ $L_{n}\left(g_{n}\right) \|_{L^{p}}$, and the Hausdorff-Young inequality to $\left\|g_{n}-S_{n}\left(g_{n}\right)\right\|_{L^{p}}$, we can obtain the following lemma in the case of $2<p<\infty$.

Lemma 2. Let $2<p<\infty$. Then there exists a trigonometric polynomial $g_{n}^{\star}(x)$ such that

$$
\begin{gathered}
\left\|g_{n}^{\star}-L_{n}\left(g_{n}^{\star}\right)\right\|_{L^{p}} \geqslant c_{2} \lambda_{n}^{-t} n^{1 / 2} \\
\left\|g_{n}^{\star}-S_{n}\left(g_{n}^{\star}\right)\right\|_{L^{p}}=0\left(\lambda_{n}^{-t / q} n^{1 / q}\right), \quad 1 / p+1 / q=1 .
\end{gathered}
$$

Proof of the thoerem: First suppose that $1<p \leqslant 2$. Let $n_{i}=8$, select $n_{j+1}$ such that

$$
\lambda_{n_{j+1}} \leqslant \lambda_{n_{j}}^{2} \text { and } n_{j+1} \geqslant \lambda_{n_{j}}^{-3}
$$

Define $f(x)$ by

$$
f(x)=\sum_{j=1}^{\infty} \lambda_{n_{j}}^{n_{j}} g_{n_{j}}(x) .
$$

It is clear that $f \in C_{2 \pi}$ is infinitely differentialble. Minkowski's inequality implies that

$$
\begin{aligned}
& \left\|f-L_{n_{k}}(f)\right\|_{L^{p}} \geqslant \lambda_{n_{k}}^{n_{k}}\left\|g_{n_{k}}-L_{n_{k}}\left(g_{n_{k}}\right)\right\|_{L^{p}} \\
& \quad-\sum_{j=k+1}^{\infty} \lambda_{n_{j}}^{n_{j}} \sum_{m=1}^{\left[\left.\lambda_{n}^{-3}\right|^{m\left(2 n_{j}+1\right)+n_{j}-1}\right.} \sum_{l=m\left(2 n_{j}+1\right)}^{m}\left\|\cos l x-L_{n_{k}}(\cos l t, x)\right\|_{L^{p}},
\end{aligned}
$$


so, by (3) and (4),

(8) $\left\|f-L_{n_{k}}(f)_{L^{p}}\right\| \geqslant c_{6} \lambda_{n_{k}}^{n_{n^{-}}-3} n_{k}^{1 / q}-0\left(\sum_{j=k+1}^{\infty} \lambda_{n_{j}}^{n_{j}-4}\right) \geqslant c_{6} \lambda_{n_{k}}^{n_{k}-3} n_{k}^{1 / q}-0\left(\lambda_{n_{k}}^{n_{k}}\right)$.

At the same time, from (5),

(9) $\left\|f-S_{n_{k}}(f)\right\|_{L^{p}} \leqslant \sum_{j=k}^{\infty} \lambda_{n_{j}}^{n_{j}}\left\|g_{n_{j}}-S_{n_{k}}\left(g_{n_{j}}\right)\right\|_{L^{p}}=0\left(\lambda_{n_{k}}^{n_{k}-3 / 2} n_{k}^{1 / 2}\right)=0\left(\lambda_{n_{k}}^{n_{k}-2}\right)$.

Combining (8) and (9) we get, for sufficiently large $k$,

$$
\frac{\left\|f-L_{n_{k}}(f)\right\|_{L P}}{\left\|f-S_{n_{k}}(f)\right\|_{L^{p}}} \geqslant c_{\tau} \lambda_{n_{k}}^{-1} n_{k}^{1 / q}
$$

thus in this case the theorem is proved. For the case $2<p<\infty$, taking $t=3 p / 2$ and starting from (6) and (7), we can construct the function required in a similar way. The proof of the theorem is coinpleted.

In $L^{1}$ space there is also such a "bad result"; we will discuss it in another paper using a different method of construction.

\section{REFERENCES}

[1] Gongji Feng, 'Asymplotic expansion of the Lebesque constants associated with trigonometric interpolation corresponding to the equidistant nodal points', Chinese, Math. Numer. Sinica 7 (1985), 420-425.

[2] A. Zygmund, Trigonometric Series (Cambridge University Press, Cambridge, 1959).

\section{Dalhousie University}

Department of Math Stats and Computer Science

Halifax

Nova Scotia

Canada B3H 3J5 